\title{
INDEPENDANT POWER SUPPLY SOURCE FOR THE STATION OF CATHODIC PROTECTION OF PIPELINES AGAINST CORROSION
}

\author{
Vladimir Sergeevich Ezhov ${ }^{1^{\star}}$ Natalia Evgenievna Semicheva ${ }^{1}$ Sergey Vladimirivich Berezin ${ }^{1}$ \\ Veronika Vitalievna Makhova ${ }^{1}$ Alexey Petrovich Burtsev ${ }^{1}$ Artem Viktorovich Brezhnev $^{1}$ \\ Roman Sergeevich Drozhzhin ${ }^{1}$
}

${ }^{1}$ Southwest State University, Kursk, Russia

The paper deals with the issues of direct conversion of thermal energy to electric one due to the use of the thermoelectricity effect to provide independent operation of a cathodic protection station when heat transfer fluids flow through pipelines. The experimental setup description, applied techniques and procedure, and the experimental results are presented. A possibility of generating electricity using thermoelectricity effect for a cathodic protection station independent power supply source is demonstrated.

Key words: Electrochemical corrosion, Thermoelectricity, Thermal energy, Power supply source, Pipeline, Heat transfer fluid, Fluid, Gas, Voltage, Current intensity, Capacity, Cathodic protection station

\section{INTRODUCTION}

The most common method of protecting equipment (including pipelines) made of carbon, low- and high-alloy and high-chromium steels, tin, zinc, copper and copper-nickel alloys, aluminum, lead, titanium and their alloys from electrochemical corrosion is cathodic protection (protection by means of external current). It is based on imposing a negative potential on a piece to be protected. In this case, pipelines are equipped with cathodic protection stations (CPS). To function, such a station requires an external power supply source, which increases the cost for CPS design and operation, especially in remote and inaccessible areas of pipeline location. At the same time, it is known that a significant part of pipelines is intended to be used for transporting liquids and gases with relatively high temperatures $\left(100^{\circ} \mathrm{C}\right.$ and higher), whereas the surrounding medium (ground or water) can have a temperature close to zero and lower. The latter circumstance indicates the possibility of generating thermoelectricity on such pipelines based on using a considerable temperature difference between the working medium and the environment.

The essence of the phenomenon of thermoelectricity (Seebeck effect) is that when the ends of a circuit consisting of two dissimilar metal materials, whose joints are at different temperatures, are closed, an electromotive force (EMF) emerges in the circuit.

Thermoelectricity generation is one of the promising, and in some cases the only available way of direct conversion of thermal energy into electrical one. It is especially important that with such a transformation there is no intermediate phase, when thermal energy is first transformed into mechanical one, and only after that mechanical energy is transformed into electrical [01-03].

\section{PROPOSED ENGINEERING SOLUTIONS}

Based on the use of the thermoelectricity effect, engineering proposals of designing an independent power supply source for CPS were developed at the Department of Heat, Gas and Water Supply of the Southwest State University (Kursk, Russia) [04-06]. Figure 1 demonstrates the diagram of one of the engineering proposals [06].

The EMF source under consideration works as follows. After filling the pipeline 3 and after gas (liquid) therein begins flowing, heat exchange between gas (liquid) in the pipe and surrounding ground (water) takes place. For example, the gas (liquid) temperature $t_{\Pi}$ is lower than the temperature $t_{c}$ of the soil (water) that is in contact with the outer surface of the EMF source 1, which is made of a hydrostatically dielectric high-conductivity material; due to the temperature difference $t_{\Pi}-t_{c}$ heat exchange between cold gas (liquid) flowing through the pipe 4 and the surrounding ground (water) takes place; the areas of heating and cooling heat up or cool down; the areas of heating and cooling consist of a layer of material inside which soldered two-layer flattened ends of the TIC 15 made of metals M1 and M2 and arranged in parallel to the surface of the pipe 3 in the area of semi-rings 9 and 10 and in parallel to the longitudinal ribs 11 . The design of the two-layer ends of the TIC 15 connected to each other (e.g., by means of soldering) allows increasing the area of heating and cooling between the surfaces of the semi-rings 9, 10 and the ribs 11 .

As a result of heat exchange processes, a temperature difference is created between the soldered two-layer, flattened, tightly pressed together ends of the TIC 15 made of metals M1 and M2 located at the edges of the ribs 11 and opposite ends of the same metal segments M1 and M2 located in the semi-rings 9 and 10 . 
The created temperature difference between the heating and cooling areas causes electron emission in all TICs 15 and, accordingly, thermoelectricity generation in the zigzag rows of TES 14. At the required voltage and current, on the command from the control unit 5, the generated thermoelectricity is supplied to the protected pipeline section 3 through the current leads 16, the regulating block 2 , and the connecting cable 6 .

The magnitude of the difference between the electric potential and the current intensity at the current leads 16 of the power supply source (EMF) 1 depends on the temperature difference at the soldered joints of metals $M 1$ and $M 2$, their characteristics, and the number of the TIC 15 in the TES 14. When necessary, several power supplies sources 1 can be installed. Depending on the gas (liquid) flow rate and the temperature difference $\left(t_{\Pi}-\right.$ $t_{c}$ ), the required voltage $U$ and current $I$ are controlled by the commands from the control unit 5 to the regulating block 2, which turns off or on the TES 14 in power supply source 1.

Figure 1: Device for thermoelectric protection of pipelines against corrosion: 1 - power supply source (EMF);

2 - regulating block; 3 - section of the pipeline to be protected; 4 - anodic groundbed; 5 - control unit; 6 ,

7, 8 - connecting cables; 9, 10 - semi-rings of power supply source; 11, 12, 13 - longitudinal ribs and flanges with fastening holes; 14 - zigzag rows of thermoelectric sections (TESs); 15- thermionic converters (TIC); 16 - current leads
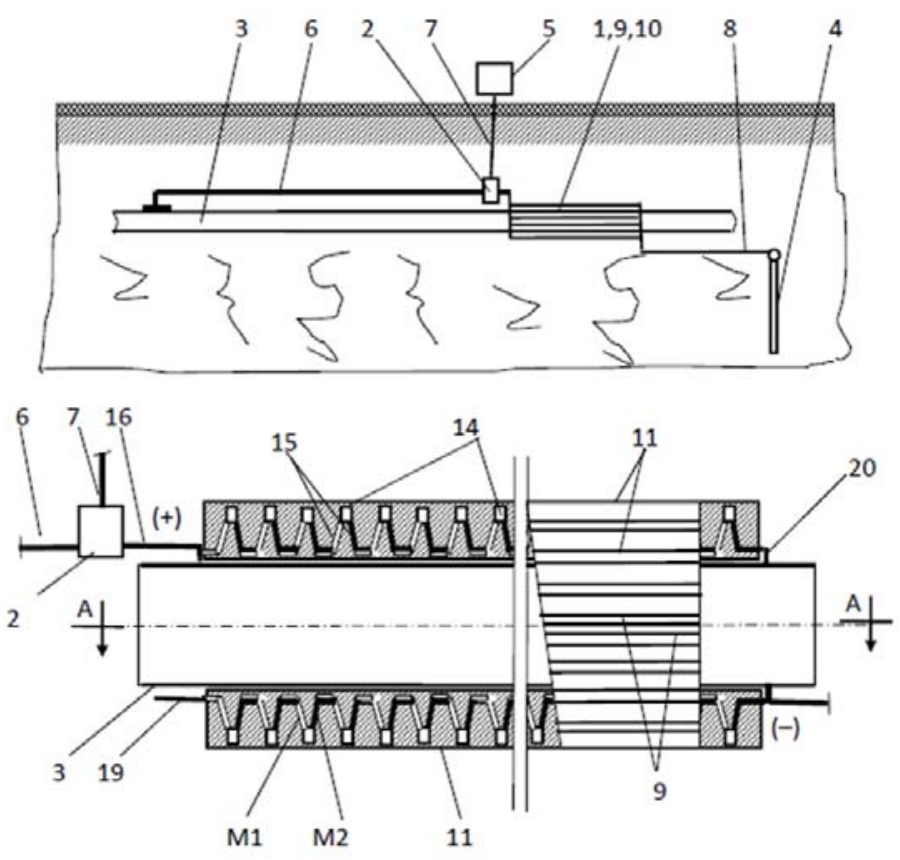

$V=w \cdot f$

where $w$ is the hot air velocity at the outlet of the pipe, $\mathrm{m} / \mathrm{sec}$;

$f$ is the free flow cross section of the pipe, $\mathrm{m}^{2}$. 
Heat content of the air passing through the pipe was found from the formula:

$$
\mathrm{Q}_{1}=\mathrm{V} \cdot \mathrm{c}_{\mathrm{p}} \cdot\left(\mathrm{t}_{\mathrm{H}}-\mathrm{t}_{\mathrm{K}}\right)
$$

where $c_{p}$ is the mean heat capacity of the air, $\mathrm{kJK} / \mathrm{m}^{3 .}{ }^{\circ} \mathrm{C}$; $t_{H}$ is the temperature of the air at the inlet of the pipe, ${ }^{\circ} \mathrm{C}$; $t_{\kappa}$ is the temperature of the air at the outlet of the pipe, ${ }^{\circ} \mathrm{C}$. Heat losses through the pipe surface were determined as follows:

$$
\mathrm{Q}_{2}=\mathrm{c}_{\mathrm{p}} \cdot \Delta \mathrm{t}
$$

The efficiency of the power source 1 (the coefficient of the direct conversion of thermal energy into electrical one) was determined according to the formula:

$$
\eta=P / Q_{2}
$$

where $P$ is the power of the power (EMF) supply source, W.

The results of the experiment are given in Table 1 (the denominator is the values of one section, the numerator is the totalized indications of the EMF source).

The experiment when the EMF source was located on the surface of the pipe showed a lower effectiveness of thermoelectricity production, which can be explained by the fact that the outer surface of the pipe was in a motionless air environment, whereas at the first stage of the experiment, the outer surface of the TES was blown with air from the heat gun. Just like in the first case, about $1 \%$ of the total heat transferred through the heat exchange surface was consumed to generate electrical energy.

The obtained results show that when the temperature difference between the pipe surface and the external environment is equal to $(100-125){ }^{\circ} \mathrm{C}$, the use of the thermoelectricity effect for an independent EMF source of the cathodic protection station is completely justified, which can be demonstrated by an example.

The output energy parameters of cathodic protection stations (CPSs) produced in Russia for pipelines with a diameter greater than $400 \mathrm{~mm}$ are as follows: rated output voltage is $50 \mathrm{~V}$; rated output current is $60 \mathrm{~A}$; rated output power is 3,000 W [09].

The calculation (without taking into account the resistances) using the above experimental data, e.g. at $\Delta \mathrm{t}=$ $125^{\circ} \mathrm{C}$, shows that to obtain voltage $\mathrm{U}=50 \mathrm{~V}$, a section with a TIC number $n=12,000$ pcs is required; it will provide current intensity of $9.2 \mathrm{~A}$; and to obtain a current intensity of $50 \mathrm{~A}$ and power of $\mathrm{P}=3,000 \mathrm{~W}$, the total number of TICs will be $n_{\text {total }}=65,600$ pcs. The required heat exchange surface of the power source for the CPS will be $(10-20) \mathrm{m}^{2}$; it depends on the pitch between the TICs in the row and the pitch from the TIC's rows, its length depends on the diameter of the pipeline. So, for example, for a pipeline with the diameter of $500 \mathrm{~mm}$ and the area of the EMF source heat exchange surface of 15 $\mathrm{m}^{2}$, the length of the power source (EMF) will be $10 \mathrm{~m}$.

Table 1: The results of the 2 nd stage of the experiment

\begin{tabular}{|c|c|c|c|c|c|}
\hline No & $\begin{array}{c}\text { Temperature of the } \\
\text { pipe surface, } t_{T}^{\circ} \mathrm{C}\end{array}$ & $\begin{array}{c}\text { Temperature } \\
\text { difference, } \\
\Delta t^{\circ} \mathrm{C}\end{array}$ & $\begin{array}{c}\text { Voltage } \\
V, \mathrm{~V}\end{array}$ & $\begin{array}{c}\text { Current intensity } \\
I, \mathrm{~mA}\end{array}$ & $\begin{array}{c}\text { Power } \\
P, \mathrm{~W}\end{array}$ \\
\hline 1 & 100 & 75 & $0.06 / 0.5$ & $10 / 80$ & $0.006 / 0.048$ \\
\hline 2 & 110 & 85 & $0.065 / 0.55$ & $12 / 96$ & $0.0078 / 0.0625$ \\
\hline 3 & 120 & 95 & $0.091 / 0.7$ & $14 / 100$ & $0.0127 / 0.07$ \\
\hline 4 & 130 & 105 & $0.1 / 0.8$ & $16 / 120$ & $0.016 / 0.096$ \\
\hline 5 & 140 & 115 & $0.11 / 0.9$ & $20 / 155$ & $0.022 / 0.14$ \\
\hline 6 & 150 & 125 & $0.12 / 1.0$ & $25 / 185$ & $0.028 / 0.185$ \\
\hline 7 & 160 & 135 & $0.135 / 1.1$ & $30 / 215$ & $0.0406 / 0.237$ \\
\hline 8 & 170 & 145 & $0.14 / 1.2$ & $36 / 240$ & $0.0505 / 0.29$ \\
\hline 9 & 180 & 155 & $0.15 / 1.3$ & $38 / 272$ & $0.0572 / 0.354$ \\
\hline 10 & 190 & 165 & $0.16 / 1.42$ & $39 / 295$ & $0.062 / 0.427$ \\
\hline 11 & 200 & 175 & $0.165 / 1.5$ & $40 / 306$ & $0.066 / 0.465$ \\
\hline 12 & 210 & 185 & $0.17 / 1.6$ & $42 / 320$ & $0.0713 / 0.51$ \\
\hline
\end{tabular}




\section{CONCLUSION}

A significant number of cathodic stations for protection of pipelines from electrochemical corrosion can be provided with independent power supplies (EMF).

The considered engineering solutions and experimental data show that the thermoelectricity effect can be used to develop an independent power source for the cathodic protection station in pipelines when transporting heat transfer fluids have a temperature of above $100^{\circ} \mathrm{C}$.

Thermo emission converters consisting of paired wire segments made of metals M1- chromel and M2 - copel can be used as the main structural element in independent power supply sources of cathodic stations.

\section{ACKNOWLEDGMENTS}

The research was carried out within the framework of the state task for 2017 in the field of scientific activities "Research and development of the design and structure of an independent EMF source for cathodic protection station (13.8087.2017 / BCh)

\section{REFERENCES}

1. Ioffe, A. F., Stilbans, L. S., lordanishvili, E. K., Stavitskaya, T. S. Thermoelectric cooling. M.-L .: Izd. AN SSSR, 1956.

2. Burshtein, A. I. Physical foundations for the calculation of semiconductor thermoelectric devices. Moscow: Fizmatgiz, 1962.

3. Goldsmid, H. J. Applications of thermoelectricity. (Methuen's monographs on physical subjects). London: Methuen \& Co. Ltd.; New York, Wiley, 1960.

4. Patent of the Russian Federation No 2509266. Thermoelectric link for pipe / Yezhov, V. S., Bul. No 7, P. 8, 2014.

5. Patent of the Russian Federation No 2510434. Device for thermoelectric protection of the pipeline against corrosion / Yezhov, V. S., Berezin, S. V., Bul. No 9, P. 8. 2014

6. Patent of the Russian Federation No 2550073, Device for thermoelectric protection of the pipeline against corrosion / Yezhov, V. S., Emelyanov, S. G., Semicheva, N. E., Berezin, S. V., Panin, A. A., Burtsev, A. P., Soshnikova, A. I., Tsukanova, D. V., Bul. No 13, P. 10, 2015.

7. Yezhov, V. S., Semicheva, N. E. / Application of low-potential thermal energy for buildings power supply / Proceedings of Southwest State University. -Kursk, №1, 2012. P.56-62.
8. Ezhov, V. S., Semicheva, N. E., Kosinov, A. V., Lysenko, I. V., Pivovarov, A. S. Integrated application of solar energy and thermoelectric effect for buildings power supply / Bulletin of the central regional branch. Russian Academy of Architecture and Building Sciences. - Kursk - Voronezh, vol. 12, 2013. C.150-156.

9. Semenov, A. G., Sysa, L. P. What is electrochemical protection and how to choose a cathodic station / Heat supply news, No 10 (50), 2004. 